\title{
Exp-Function Method with Computer Symbolic Computation for Exact Solutions of A Nonlinear Differential Equation
}

\author{
Sheng Zhang ${ }^{1, a}$ and Dong-Dong Liü,b \\ ${ }^{1}$ School of Mathematics and Physics, Bohai University, Jinzhou 121013, PR China \\ aszhangchina@126.com, ${ }^{b} 461922571 @ q q . c o m$
}

Keywords: Exact solution; Nonlinear differential equation; Exp-function method

\begin{abstract}
In this paper, the exp-function method is generalized to solve a first-order nonlinear differential equation with forth-degree nonlinear term. As a result, three new exact solutions with free parameters are obtained, the first two of which include generalized hyperbolic function solutions and generalized trigonometric solutions as special cases and the third one is a rational solution. It is shown that the generalized exp-function method with the help of computer symbolic computation provides a straightforward and effective mathematical tool for solving nonlinear differential equations.
\end{abstract}

\section{Introduction}

Since the celebrated Korteweg-de Vries (KdV) equation was solved in 1965 [1], exactly solving nonlinear differential equations (DEs) has attached much attentions [2-12]. With the development of computer science, recently, directly searching for exact solutions of nonlinear DEs becomes possible. This is due to some computer symbolic computation systems like Mathematica or Maple, which enable us to perform the complex and tedious computation on computers [13-20].

Recently, we proposed a generalized exp-function method [21] to solve fractional DEs by introducing the following new and more general ansätz:

$$
u(\xi)=\frac{\sum_{n=-c}^{d} a_{n}\left[\mu E_{\alpha}\left(n \lambda \xi^{\alpha}\right)+\rho \xi^{\alpha}\right]^{n}}{\sum_{m=-p}^{q} b_{m}\left[\mu E_{\alpha}\left(m \lambda \xi^{\alpha}\right)+\rho \xi^{\alpha}\right]^{m}},
$$

where $\mu=\{0,1\}$ and $\rho=\{0,1\}$ are two embedded constants, $E_{\alpha}\left(\xi^{\alpha}\right)$ is the Mittag-Leffler function defined as [22]:

$$
E_{\alpha}(z)=\sum_{k=0}^{\infty} \frac{z^{k}}{\Gamma(1+k \alpha)} .
$$

The present paper is motivated by the desire to extend the generalized exp-function method [21] to construct exact solutions of a first-order nonlinear ordinary differential equation (ODE) with forth-degree nonlinear term:

$$
\left[{ }_{0} D_{\xi}^{\alpha} z(\xi)\right]^{2}=a z^{2}(\xi)+b z^{3}(\xi)+c z^{4}(\xi), 0<\alpha \leq 1,
$$

where ${ }_{0} D_{\xi}^{\alpha}$ is the modified Riemann-Liouville derivative with respective to $\xi$, which has some useful properties [23-25], $a, b$ and $c$ are real parameters.

\section{Exact solutions}

To solve Eq. (3), we firstly use ansätz (1) to balance the linear term of highest (lowest) order of the Mittag-Leffler function in Eq. (2) with the highest (lowest) order nonlinear term, we have $p=c$ and $q=d$. We can freely choose the values of $c$ and $d$, but the final solution does not strongly depend 
upon the choice of values of $c$ and $d$. For simplicity, we choose $p=c=1, d=q=1$, then ansätz (1) becomes:

$$
z(\xi)=\frac{\sum_{n=-1}^{1} a_{n}\left[\mu E_{\alpha}\left(n \lambda \xi^{\alpha}\right)+\rho \xi^{\alpha}\right]^{n}}{\sum_{m=-1}^{1} b_{m}\left[\mu E_{\alpha}\left(m \lambda \xi^{\alpha}\right)+\rho \xi^{\alpha}\right]^{m}} .
$$

Secondly, substituting Eq. (4) into Eq. (3) and equating to zero of the coefficients of all powers of $\left(\xi^{\alpha}\right)^{i}\left[E_{\alpha}\left(\lambda \xi^{\alpha}\right)\right]^{j}(i, j=0,1,2, \cdots, 8)$ yields a set of algebraic equations for $a_{1}, a_{0}, a_{-1}, b_{1}, b_{0}, b_{-1}, \lambda, \mu$ and $\rho$. If we set $\mu=1$ and $\rho=0$, the set of algebraic equations can be simplified. Solving the set of simplified algebraic equations with the help of Mathematica, we obtain two cases as follows:

$$
a_{1}=0, a_{0}=-\frac{2 a b_{0}}{b}, a_{-1}=0, b_{1}=b_{1}, b_{0}=b_{0}, b_{-1}=\frac{b_{0}^{2}\left(b^{2}-4 a c\right)}{4 b^{2} b_{1}}, \lambda= \pm \sqrt{a} .
$$

and

$$
a_{1}=-2 a, a_{0}=0, a_{-1}=0, b_{1}=b, b_{0}=0, b_{-1}=b_{-1}, \lambda= \pm \frac{\sqrt{a}}{2}, b^{2}-4 a c=0 .
$$

If we set $\mu=0$ and $\rho=1$, the set of algebraic equations can be simplified to another one. Solving the set of simplified algebraic equations with the help of Mathematica, we have

$$
a_{1}=0, a_{0}= \pm \frac{b_{1} \sqrt{c} \Gamma(1+\alpha)}{c}, a_{-1}=0, b_{1}=b_{1}, b_{0}=b_{0}, b_{-1}=0, \lambda=\lambda, a=0, b=0 \text {. }
$$

We, therefore, from Eqs. (4)-(6) obtain the following two exact solutions of Eq. (3):

$$
\begin{aligned}
& z(\xi)=\frac{-\frac{2 a b_{0}}{b}}{b_{1} E_{\alpha}\left( \pm \sqrt{a} \xi^{\alpha}\right)+b_{0}+\frac{b_{0}^{2}\left(b^{2}-4 a c\right)}{4 b^{2} b_{1}} E_{\alpha}\left(\mp \sqrt{a} \xi^{\alpha}\right)}, \\
& z(\xi)=\frac{-2 a E_{\alpha}\left( \pm \frac{\sqrt{a}}{2} \xi^{\alpha}\right)}{b E_{\alpha}\left( \pm \frac{\sqrt{a}}{2} \xi^{\alpha}\right)+b_{11} \exp E_{\alpha}\left(\mp \frac{\sqrt{a}}{2} \xi^{\alpha}\right)}, b^{2}-4 a c=0 .
\end{aligned}
$$

At the same time, Eqs. (4) and (7) arrive at a rational solution of Eq. (3):

$$
z(\xi)= \pm \frac{b_{1} \sqrt{c} \Gamma(1+\alpha)}{c\left(b_{0}+b_{1} \xi^{\alpha}\right)} .
$$

If we set $b_{0}=\mp 2, b_{1}=\sqrt{b^{2}-4 a c} / b$ and $b^{2}-4 a c>0$, then Eq. (8) gives a generalized hyperbolic function solution and a generalized trigonometric function solution:

$$
\begin{aligned}
& z(\xi)=\frac{ \pm 2 a \operatorname{sech}_{\alpha}(\sqrt{a} \xi)}{\sqrt{b^{2}-4 a c} \mp b \operatorname{sech}_{\alpha}(\sqrt{a} \xi)}, b^{2}-4 a c>0, a>0, \\
& z(\xi)=\frac{ \pm 2 a \sec _{\alpha}(\sqrt{-a} \xi)}{\sqrt{b^{2}-4 a c} \mp b \sec _{\alpha}(\sqrt{-a} \xi)}, b^{2}-4 a c>0, a<0 .
\end{aligned}
$$

If we set $b_{0}=-2, b_{1}=\sqrt{4 a c-b^{2}} / b$ and $b^{2}-4 a c<0$, then Eq. (9) becomes: 


$$
\begin{aligned}
& z(\xi)=\frac{ \pm 2 a \operatorname{csch}_{\alpha}(\sqrt{a} \xi)}{\sqrt{4 a c-b^{2}} \mp b \operatorname{csch}_{\alpha}(\sqrt{a} \xi)}, b^{2}-4 a c<0, a>0, \\
& z(\xi)=\frac{\mp 2 a i \csc _{\alpha}(\sqrt{-a} \xi)}{\sqrt{4 a c-b^{2}} \pm b i \csc _{\alpha}(\sqrt{-a} \xi)}, b^{2}-4 a c<0, a<0 .
\end{aligned}
$$

If we set $b_{11}=b$, then Eq. (9) becomes

$$
\begin{aligned}
& z(\xi)=-\frac{a}{b}\left[1 \pm \tanh _{\alpha}\left(\frac{\sqrt{a}}{2} \xi\right)\right], b^{2}-4 a c=0, a>0, \\
& z(\xi)=-\frac{a}{b}\left[1 \pm i \tan _{\alpha}\left(\frac{\sqrt{-a}}{2} \xi\right)\right], b^{2}-4 a c=0, a<0 .
\end{aligned}
$$

If we set $b_{11}=-b$ then Eq. (9) becomes

$$
\begin{aligned}
& z(\xi)=-\frac{a}{b}\left[1 \pm \operatorname{coth}_{\alpha}\left(\frac{\sqrt{a}}{2} \xi\right)\right], b^{2}-4 a c=0, a>0, \\
& z(\xi)=-\frac{a}{b}\left[1 \mp i \cot _{\alpha}\left(\frac{\sqrt{-a}}{2} \xi\right)\right], b^{2}-4 a c=0, a<0 .
\end{aligned}
$$

To express the solutions obtained above, we have used the following generalized hyperbolic functions and generalized trigonometry functions defined by the Mittag-Leffler function [21]:

$$
\begin{aligned}
& \sin _{\alpha}\left(x^{\alpha}\right)=\frac{E_{\alpha}\left(i x^{\alpha}\right)-E_{\alpha}\left(-i x^{\alpha}\right)}{2 i}, \cos _{\alpha}\left(x^{\alpha}\right)=\frac{E_{\alpha}\left(i x^{\alpha}\right)+E_{\alpha}\left(-i x^{\alpha}\right)}{2}, \tan _{\alpha}(x)=\frac{\sin _{\alpha}(x)}{\cos _{\alpha}(x)}, \\
& \cot _{\alpha}(x)=\frac{\cos _{\alpha}(x)}{\sin _{\alpha}(x)}, \sec _{\alpha}(x)=\frac{1}{\cos _{\alpha}(x)}, \csc _{\alpha}(x)=\frac{1}{\sin _{\alpha}(x)}, \\
& \sinh _{\alpha}(x)=\frac{E_{\alpha}\left(x^{\alpha}\right)-E_{\alpha}\left(-x^{\alpha}\right)}{2}, \cosh _{\alpha}(x)=\frac{E_{\alpha}\left(x^{\alpha}\right)+E_{\alpha}\left(-x^{\alpha}\right)}{2}, \\
& \tanh _{\alpha}(x)=\frac{\sinh _{\alpha}(x)}{\operatorname{coth}_{\alpha}(x)}, \operatorname{coth}_{\alpha}(x)=\frac{\cosh _{\alpha}(x)}{\sinh _{\alpha}(x)}, \operatorname{sech}_{\alpha}(x)=\frac{1}{\cosh _{\alpha}(x)}, \operatorname{csch}_{\alpha}(x)=\frac{1}{\sinh _{\alpha}(x)} .
\end{aligned}
$$

We note here that when $\alpha \rightarrow 1$, solutions (10)-(18) change into the ones of the standard form nonlinear ODE [26].

\section{Conclusion}

In summary, we have generalized the exp-function method with computer symbolic computation to solve, in a uniformed way, the first-order nonlinear ODE with forth-degree nonlinear term by introducing the algorithm devised in this paper. As a result, three exact solutions with free parameters are obtained, from two ones of which the generalized hyperbolic function solutions, generalized trigonometry function solutions are also derived when setting the free parameters as special values. To the best of our knowledge, these solutions have not been obtained in literature. The generalized exp-function method can be also applied to other nonlinear fractional ODEs. How to employ the first-order nonlinear ODE (1) with its exact solutions as the subsidiary equation to solve more types of fractional partial differential equations (PDEs) arising in practical problems is worthy of study. This is our tasks in the future. 


\section{Acknowledgements}

This work was supported by the Natural Science Foundation of Educational Committee of Liaoning Province (L2012404) of China, the PhD Start-up Funds of Bohai University (bsqd2013025) and Liaoning Province of China (20141137), the Liaoning BaiQianWan Talents Program (2013921055) and the Natural Science Foundation of China (11371071).

\section{References}

[1] C.S. Garder, J.M. Greene M.D. Kruskal and R.M. Miura: Phys. Rev. Lett. Vol. 19 (1965), p. 1095

[2] M.R. Miurs: Bäcklund Transformation (Springer-Verlag, Berlin 1978)

[3] R. Hirota: Phys. Rev. Lett. Vol. 27 (1971), p. 1192

[4] C.S. Garder, J.M. Greene, M.D. Kruskal and R.M. Miura: Comm. Pure Appl. Math. Vol. 27 (1974), p. 97

[5] V.E. Zakharov and A.B. Shabat: Sov. Phys. JETP Vol. 34 (1972), p. 62

[6] M. Wadati: J. Phys. Soc. Jpn. Vol. 32 (1973), p. 1289

[7] M.J. Ablowitz, D.J. Kaup, A.C. Newell and H. Segur: Phys. Rev. Lett. Vol. 30 (1973), p. 1262

[8] H. Flaschka: Prog. Theor. Phys. Vol. 51 (1974), p. 703

[9] M.J. Ablowitz and J.F. Ladik: J. Math. Phys. Vol. 16 (1975), p. 598

[10]A.I. Nachman and M.J. Ablowitz: Stud. Appl. Math. Vol. 71 (1984), p. 243

[11]M.J. Ablowitz and P.A. Clarkson: Soliton, Nonlinear Evolution Equations and Inverse Scattering, (Cambridge University Press, Cambridge 1991)

[12]M.L. Wang: Phys. Lett. A Vol. 213 (1996), p. 279

[13]E.G. Fan: Phys. Lett. A Vol. 300 (2002), p. 243

[14]S. Zhang and T.C. Xia: Commun. Theor. Phys. Vol. 45 (2006), p. 985

[15]S. Zhang, Q. Gao, Q.A. Zong and D. Liu: Therm. Sci. Vol. 16 (2012). p. 1576

[16]S. Zhang, Q.A. Zong, Q. Gao and D. Liu: J. Nano Res-SW Vol. 23 (2013), p. 113

[17]S. Zhang, B. Xu and A.X. Peng: Appl. Mech. Mater. Vol. 394 (2013), p. 571

[18]S. Zhang, Y.Y. Zhou and B. Cai: Adv. Mater. Res. Vol. 989-994 (2014), p. 1716

[19]S. Zhang and B. Cai: Nonlinear Dyn. Vol. 78 (2014), p. 1593

[20]S. Zhang and D. Wang: Therm. Sci. Vol. 18 (2014), p. 1555

[21]S. Zhang: Nonlinear Sci. Lett. A Vol. 1 (2010), p. 143

[22]I. Podlubny: Fractional Differential Equations: An Introduction to Fractional Derivatives, Fractional Differential Equations, to Methods of Their Solution and Some of Their Applications (Academic Press, New York 1999)

[23]G. Jumarie: Comput. Math. Appl. Vol. 51 (2006), p. 1367

[24]G.C. Wu and E.W.M. Lee: Phys. Lett. A Vol. 374 (2010), p. 2506

[25]G.C. Wu and J.H. He: Nonlinear Sci. Lett. A Vol. 3 (2010), p. 281

[26]S. Zhang, J.L. Tong and W. Wang: Comput. Math. Appl. Vol. 58 (2009), p. 2294 